the relationship between community treatment orders and readmission rates is of a different complexity than that between chemotherapy and cancer remission, or between digitalis and cardiac function.

We acknowledge, and celebrate, the contribution of RCTs to evidence-based healthcare. But there remains a need for a plurality of methods. However astute and research-literate the clinician, RCTs select participants in ways that can make generalisation to real-world settings difficult. Realist approaches that help bridge the gap between the 'what' and the 'how' of clinical outcomes can only be a good thing. And the more complex the intervention and the more context dependent - the more important this is. For us, RCTs alone are unlikely to be sufficient.

Parity of esteem for psychiatry is undoubtedly worthwhile, but this does not mean we have to imitate other specialities; as so often in the past, we can lead the way instead. Primus inter pares.

1 Pawson R. The Science of Evaluation. Sage, 2013.

2 Kisely S, Hall K. An updated meta-analysis of randomized controlled evidence for the effectiveness of community treatment orders. Can J Psychiatry 2014; 59: 561-4.

3 Subramanian SV, Kim R, Christakis NA. The "average" treatment effect: a construct ripe for retirement. A commentary on Deaton and Cartwright. Soc Sci Med 2018; 210: 77-82.

Scott Weich, Head of the Mental Health Research Unit, Honorary Consultant Psychiatrist and Professor of Mental Health, School of Health and Related Research, University of Sheffield, UK; Craig Duncan, Senior Research Fellow, Department of Geography, University of Portsmouth, UK; Sarah-Jane Fenton, Lecturer in Mental Health Policy, Institute for Mental Health, Warwick University, UK; Graham Moon, Professor of Spatial Analysis in Human Geography, School of Geography and Environment, University of Southampton, UK: Swaran Singh, Head of Mental Health and Wellbeing, Warwick Medical School, Warwick University, UK; Kamaldeep Bhui, Professor of Cultural Psychiatry and Epidemiology, Centre for Psychiatry, Barts and The London School of Medicine \& Dentistry, Queen Mary University, UK; Liz Twigg, Professor of Human Geography, Department of Geography, University of Portsmouth, UK; Jason Madan, Professor in Health Economics, Warwick Clinical Trials Unit and Director of Postgraduate Research, Warwick Medical School, Warwick University, UK; David Crepaz-Keay, Head of Empowerment and Social Inclusion, Mental Health Foundation, UK; Helen Parsons, Senior Research Fellow, Warwick Medical School, Warwick University, UK.

Email: s.weich@sheffield.ac.uk

doi:10.1192/bjp.2018.217

\section{Does pharmacotherapy really have as enduring effects as psychotherapy in anxiety disorders? Some doubts}

Bandelow et al recently presented a meta-analysis testing the assumption that the effects of psychotherapy in anxiety disorders are more endurable than those of pharmacotherapy. ${ }^{1}$ From nonsignificant differences between psychotherapy and pharmacotherapy in pre-follow-up effect sizes the authors concluded that '... patients who stopped taking a drug showed the same durable improvement as patients who stopped psychotherapy. ${ }^{1}$

Besides the severe (and properly discussed) limitation that an unclear percentage of patients may have started new psychological treatment or taken medications in the follow-up period, this meta-analysis raises further serious concerns.

First, the authors did not clearly specify their inclusion criteria. Apparently, they did not require head-to head comparisons of psychotherapy and pharmacotherapy as an inclusion criterion. Second, as a consequence, Bandelow et al compared pre-post and prefollow-up effect sizes of psychotherapy, medication and placebo obtained from different randomised controlled trials. Thus, the studies being compared may differ with regard to important treatment moderators such as characteristics of patient populations and setting conditions. For these and other reasons analyses of pre-post and pre-follow-up effect sizes should be avoided in meta-analyses. ${ }^{2}$

Third, Bandelow et al did not adhere to the logic of equivalence testing that includes the definition of a margin compatible with equivalence and performing two one-sided tests (TOST). ${ }^{3}$ They apparently applied the more usual two-sided superiority test. However, concluding from a non-significant two-sided superiority test that two treatments (i.e. pharmacotherapy and psychotherapy) are equally efficacious (in the long-term) is questionable. ${ }^{3}$ The traditional two-sided test and TOST often yield inconsistent results. ${ }^{4}$ Fourth, furthermore, Bandelow et al seem to have not controlled for researcher allegiance. ${ }^{5}$ Thus, a bias in favour of pharmacotherapy cannot be excluded given that the first and last authors disclose multifold collaboration with pharmaceutical companies.

Finally and of note, the authors avoid discussing potential longterm negative effects that any type of psychotropic drug treatment, particularly after long-term use, may have, for example by increasing the risk of experiencing additional psychopathological problems that do not necessarily subside with discontinuation of the drug or of modifying responsiveness to subsequent treatments. ${ }^{6}$

The data presented by Bandelow et al suggest that pharmacotherapy may have endurable effects in anxiety disorders as well. However, the authors' conclusion that in the long-term term psychotherapy and pharmacotherapy are equally efficacious in anxiety disorders is questionable for the reasons given above.

1 Bandelow B, Sagebiel A, Belz M, Görlich Y, Michaelis S, Wedekind D. Enduring effects of psychological treatments for anxiety disorders: meta-analysis of follow-up studies. Br J Psychiatry 2018; 212: 333-8.

2 Cuijpers P, Weitz E, Cristea IA, Twisk J. Pre-post effect sizes should be avoided in meta-analyses. Epidemiol Psychiatr Sci 2017; 26: 364-8.

3 Walker E, Nowacki AS. Understanding equivalence and noninferiority testing. J Gen Intern Med 2011; 26: 192-6.

4 Barker LE, Luman ET, McCauley MM, Chu SY. Assessing equivalence: an alternative to the use of difference tests for measuring disparities in vaccination coverage. Am J Epidemiol 2002; 156: 1056-61.

5 Mellers B, Hertwig R, Kahneman D. Do frequency representations eliminate conjunction effects? An exercise in adversarial collaboration. Psychol Sci 2001; 12: $269-75$.

6 Fava GA, Benas iG, Cosci F. The potential role of iatrogenic comorbidity in the interaction between pharmacotherapy and psychotherapy in anxiety disorders Verhaltenstherapie 2017; 27: 275-80.

Falk Leichsenring Professor, Psychosomatics and Psychotherapy, University of Giessen, Germany; Jürgen Hoyer, Professor, Institute of Clinical Psychology and Psychotherapy, University of Dresden, Germany. Email: Falk.Leichsenring@psycho.med. uni-giessen.de

Declaration of interest F.L. and J.H. are trained psychotherapists.

doi:10.1192/bjp.2018.225

\section{Authors' reply}

We found that gains with psychotherapy were maintained for up to 24 months. We also showed that patients who stopped medication remained stable. This is good news for the affected patients. However, as patients in the placebo groups also did not show deterioration we concluded that enduring effects observed in follow-up studies might be superimposed by spontaneous remission or effects of concurrent treatments.

For detailed inclusion criteria, we had referred to our previous meta-analysis. ${ }^{1}$ As there are only a few head-to-head follow-up comparisons of psychotherapy and pharmacotherapy, we decided to calculate pre-post effects. Thus, we were able to include as many as 93 follow-up studies, which also comprised all head-tohead comparisons.

Pre-post effect sizes do not only measure 'true' treatment effects, but also natural course and placebo effects. However, when conditions are the same in psychotherapy and pharmacotherapy studies, this comparison is fair. Patients are mainly 